\title{
Vibrissal Responses of Thalamic Cells That Project to the Septal Columns of the Barrel Cortex and to the Second Somatosensory Area
}

\author{
Hajnalka Bokor, ${ }^{1,2}$ László Acsády, ${ }^{1}$ and Martin Deschênes ${ }^{2}$ \\ ${ }^{1}$ Institute of Experimental Medicine, National Academy of Sciences, H-1450 Budapest, Hungary, and ${ }^{2}$ Centre de Recherche Université Laval Robert-Giffard, \\ Laval University, Québec City, Québec, Canada G1J 2G3
}

\begin{abstract}
The rodent somatosensory cortex contains barrel-related and septa-related circuits representing two separate streams of vibrissa information processing that differ in their response patterns and anatomical connections. Whereas barrel-related circuits process lemniscal inputs that transit through the thalamic barreloids, septa-related circuits process paralemniscal inputs and inputs that are relayed through the ventral lateral part of the ventral posterior medial nucleus (VPMvl). Septa-projecting thalamic afferents also target the secondary somatosensory cortical area. Although a number of studies have examined response properties in the lemniscal pathway, and demonstrated that barreloids receive feedback from specific sets of corticothalamic and reticular thalamic neurons, such information is currently lacking for the VPMvl. In the present study, we show that in sharp contrast to the relay cells of the barreloids VPMvl neurons exhibit large multiwhisker receptive fields that are independent of input from the principal trigeminal nucleus. Results also suggest that the topography of receptive fields and response properties in VPMvl rely on converging input from neurons of the interpolaris trigeminal nucleus. Tracer injection and single-cell labeling further reveal that the VPMvl receives input from specific populations of reticular thalamic and corticothalamic neurons. Together, these results confirm the status of the VPMvl as a thalamic relay of an independent parallel pathway of vibrissa information processing. They further indicate that a sensory pathway does not merely consist on a threeneuron chain that links the vibrissae to the cerebral cortex, but that it also involves specific sets of topographically related corticothalamic and reticular thalamic projections.
\end{abstract}

Key words: vibrissa; barrel cortex; trigeminal nuclei; ventral posterior medial nucleus; thalamus; second somatosensory area

\section{Introduction}

A large expanse of the rat primary somatosensory cortex (S1) processes vibrissal information. This cortical area comprises two intercalated cytoarchitectonic divisions: the granular zone, referred to as the barrel field, is characterized by dense cellular aggregates in layer 4; the interbarrel septa and regions surrounding the barrel field exhibit a low cell density in layer 4 and form together the dysgranular zone. The two divisions receive separate subcortical inputs, and interlaminar connections in the cortex maintain the segregation of barrel- and septa-related compartments (Kim and Ebner, 1999; Pierret et al., 2000; Shepherd and Svoboda, 2005; Alloway, 2008).

Barrels are the terminal station of the lemniscal pathway, which arises from the principal trigeminal nucleus $(\operatorname{PrV})$ and transits through the ventral posterior medial nucleus (VPM) of the thalamus. The VPM contains a single type of neurons, the relay cells, which are clustered in whisker-related aggregates called barreloids (Van der Loos, 1976; Varga et al., 2002). In the

Received Feb. 3, 2008; revised March 10, 2008; accepted March 11, 2008.

This work was supported by Canadian Institute of Health Research Grant MT-5877 to M.D.

Correspondence should be addressed to Dr. Martin Deschênes, Centre de Recherche Université Laval RobertGiffard, 2601 de la Canardière, Québec City, Québec, Canada G1J 2G3. E-mail: martin.deschenes@crulrg.ulaval.ca. DOI:10.1523/JNEUROSCI.0490-08.2008

Copyright $\odot 2008$ Society for Neuroscience $\quad$ 0270-6474/08/285169-09\$15.00/0 ventral lateral part of the VPM (VPMvl), barreloids fade out and become undistinguishable (Pierret et al., 2000; Yu et al., 2006). That part of the VPM (i.e., the VPMvl) was identified as a separate relay station of vibrissal information after anterograde tracer injection showed that it receives vibrissal input from the caudal division of the interpolaris subnucleus of the brainstem trigeminal complex (SpVic), and that it projects to the dysgranular zone of S1 and to the second somatosensory area (S2) (Pierret et al., 2000). In different studies, the VPMvl was also referred to as the tail of the barreloids (Pierret et al., 2000), or as the thalamic relay of an extralemniscal pathway (Yu et al., 2006).

A number of studies have examined vibrissal responses in the lemniscal pathway. They were performed under different types and regimens of anesthesia, and generally reported substantial similarities in response properties and receptive field size between PrV and VPM populations (Minnery et al., 2003). However, as estimated by the electrode tract reconstructions provided in some of these reports (Rhoades et al., 1987; Ito, 1988; Sugitani et al., 1990), cells in VPMvl were apparently not sampled in most of these studies. Thus, the first aim of the present study was to determine to what degree vibrissal responses in VPMvl differ from those in the barreloids, and to what degree they replicate response properties of their presynaptic partners in the SpVic (Furuta et al., 2006). The second aim was to determine whether 
VPMvl receives input from specific sets of corticothalamic and reticular thalamic (RT) neurons, as has been demonstrated for thalamic neurons of the lemniscal and paralemniscal pathways (Pinault et al., 1995; Deschênes et al., 1998; Désîlets-Roy et al., 2002).

Results indicate that receptive field structure and response properties in VPMvl derive from converging inputs of SpVic neurons, and further reveal that the VPMvl receives feedback projections from specific populations of corticothalamic and reticular thalamic neurons. This confirms the distinct status of the VPMvl as a thalamic relay for a pathway that innervates $\mathrm{S} 2$ and the septal columns of S1.

\section{Materials and Methods}

Experiments were performed in 28 male rats (250-300 g; Sprague Dawley; Charles River, St-Constant, Québec, Canada) in accordance with federally prescribed animal care and use guidelines. The Ethical Committee of Laval University approved all protocols.

Animal preparation and recording. Rats were anesthetized with ketamine $(75 \mathrm{mg} / \mathrm{kg}$ ) plus xylazine ( $5 \mathrm{mg} / \mathrm{kg})$, the left facial nerve was cut, and the animal was placed in a stereotaxic apparatus. The animals breathed freely and body temperature was kept at $37^{\circ} \mathrm{C}$ by means of a thermoregulated blanket. Throughout the experiment, a deep level of anesthesia was maintained by additional doses of anesthetics given at $1 \mathrm{~h}$ interval (ketamine $20 \mathrm{mg} / \mathrm{kg}$ plus xylazine $0.3 \mathrm{mg} / \mathrm{kg}$, i.m.). The electroencephalogram recorded in the barrel cortex displayed slow oscillations $(1-2 \mathrm{~Hz})$ that are characteristic of the stages III-3-4 described by Friedberg et al. (1999).

In different experiments, openings were made over the thalamus for recording in the VPM/VPMvl (stereotaxic coordinates: $3.3 \mathrm{~mm}$ posterior to the bregma and $3.3 \mathrm{~mm}$ lateral to the midline), or in the RT (2.8 posterior to the bregma and $3.5 \mathrm{~mm}$ lateral to the midline) (Paxinos and Watson, 1998). Single units were recorded extracellularly in the right thalamus with micropipettes $(0.5-1 \mu \mathrm{m})$ filled with a solution of potassium acetate $(0.5 \mathrm{M})$ and Neurobiotin (2\%; Vector Laboratories, Burlingame, CA). Signals were amplified, bandpass filtered $(200 \mathrm{~Hz}-3 \mathrm{kHz})$, and sampled at $10 \mathrm{kHz}$. In most experiments, cell location was assessed by the juxtacellular labeling of 1-3 units (Pinault, 1996). At the end of the experiments, rats were perfused under deep anesthesia with saline, followed by a fixative containing $4 \%$ paraformaldehyde and 0.1 or $0.5 \%$ glutaraldehyde in phosphate buffer $(0.1 \mathrm{M}, \mathrm{pH} 7.4)$. Brains were removed, cryoprotected in $30 \%$ sucrose, and cut coronally at $50-70 \mu \mathrm{m}$ on a freezing microtome or with a vibratome. Sections were processed for cytochrome oxidase (CO) and Neurobiotin histochemistry according to standard protocols that were described in detail previously (Veinante et al., 2000). Sections containing labeled cells were dehydrated and embedded in Durcupan (Fluka, Buchs, Switzerland), or mounted on gelatin coated slides, dehydrated, and covered with Depex (AMS, Abingdon, UK).

Whisker stimulation. Whiskers were cut at $2 \mathrm{~cm}$ from the skin, and the receptive field of single units was assessed by deflecting individual whiskers with a hand-held probe under a dissecting microscope. Then, the tip of the whisker was inserted into the groove of a beveled straw attached to a piezoelectric bender (Physik Instrumente, Karlsruhe, Germany) that was aligned with the axis of the hair shaft. The vibrissa was pushed in a given direction at stimulus onset, but returned passively to a neutral position at stimulus offset. Ramp-and-hold waveforms (rise/fall times, 5 ms; duration, $50 \mathrm{~ms}$; amplitude, $0.4 \mathrm{~mm}$ or $\sim 5^{\circ}$; angular velocity, $1000^{\circ} \mathrm{s}$; interstimulus interval, $1.2 \mathrm{~ms}$ ) were used to deflect vibrissae in four directions spanning $360^{\circ}$ (e.g., in $90^{\circ}$ increments relative to the horizontal alignment of the vibrissa rows). This procedure was repeated for each vibrissa that composed the receptive field of a cell. For $\sim 20 \%$ of the whiskers, the threshold for eliciting responses required deflection amplitude/velocity that exceeded the performance of the stimulator.

Data analysis. Responses to whisker deflection were collected in peristimulus time histograms (PSTHs) with a $1 \mathrm{~ms}$ bin width. For each deflection angle, the number of spikes evoked within a time window of 20 ms (for VPMvl units) or $40 \mathrm{~ms}$ (for reticular thalamic units) after stim- ulus onset was used to build polar plots of angular preference. The latency of whisker-evoked responses was estimated as the bin corresponding to 0.5 of peak value of the PSTH after three bin smoothing with a boxcar kernel. Data analysis was performed with the Neuroexplorer (Plexon, Dallas, TX), Excel (Microsoft, Redmond, WA), and OriginPro 7.5 (OriginLab, Northampton, MA) softwares.

One of the RT neurons labeled with Neurobiotin was totally reconstructed using the Neurolucida 5.2 software (MBF Bioscience, Magdeburg, Germany). The following shrinkage corrections were used: $x$-axis and $y$-axis, 1.1; $z$-axis, 2.2. These values are based on our measurements of tissue shrinkage as a result of fixation and dehydration.

Photomicrographs were taken with a Spot RT camera (Diagnostic Instruments, Sterling Heights, MI) or with an AxioCam HRC (Carl Zeiss Microimaging, Jena, Germany). Digital montages of serial photos taken through the thickness of a section were processed with the "extended depth of field function" of Image-Pro Express 6.0 (Media Cybernetics, Bethesda, MD). When necessary, brightness and contrast were adjusted using Adobe Photoshop CS2 (Adobe Systems, San Jose, CA).

PrV lesion. Parasagittal brainstem lesions were made medial to the $\operatorname{PrV}$ in three rats by passing direct current $(2.5 \mathrm{~mA}, 2 \mathrm{~s})$ through a tungsten electrode (shaft diameter, $200 \mu \mathrm{m}$; tip diameter, $50 \mu \mathrm{m}$, deinsulated over $1 \mathrm{~mm})$. Lesions were made at several frontal planes $(8.5-10 \mathrm{~mm}$ behind the bregma and $2.2 \mathrm{~mm}$ lateral to the midline) (Paxinos and Watson, 1998), and at multiple depths $(6.8-8.3 \mathrm{~mm}$ below the pia), to fully destroy the ascending axons of the PrV. In each lesioned rat the extent of the lesion was assessed on frontal sections of the brainstem stained for cytochrome oxidase.

Retrograde labeling. FluoroGold (Fluorochrome, Denver, CO) was injected iontophoretically in the VPMvl of four rats. The tracer was delivered by passing positive current pulses ( $150 \mathrm{nA}, 2 \mathrm{~s}$ duty cycle for $20 \mathrm{~min}$ ) through micropipettes (diameter; $15 \mu \mathrm{m}$ ) filled with $2 \%$ FluoroGold dissolved in cacodylate buffer $(0.1 \mathrm{M}, \mathrm{pH} 7)$. After a survival period of $2 \mathrm{~d}$, rats were perfused as above, and sections were stained for $\mathrm{CO}$ and processed for FluoroGold immunohistochemistry (rabbit anti-FluoroGold antiserum; 1:8000; Millipore Bioscience Research Reagents, Temecula, $\mathrm{CA}$; overnight, at room temperature). The antibody was revealed using a peroxidase-labeled secondary antibody (goat anti-rabbit IgG; 1:200; Millipore Bioscience Research Reagents) and nickel-3, 3 diaminobenzidine tetrahydrochloride (Ni-DAB) as a substrate.

Anterograde tracing. A micropipette (tip diameter, $20 \mu \mathrm{m}$ ) containing biotinylated dextran amine (BDA; $10 \%$ in saline) was lowered in the whisker-responsive region of S2 $(1.8 \mathrm{~mm}$ behind the bregma, $6 \mathrm{~mm}$ lateral to the midline, and $3 \mathrm{~mm}$ below the surface of the brain). The tracer was ejected with positive current pulses ( $2 \mathrm{~s}$ duty cycle, $200 \mathrm{nA}$ ) for $20 \mathrm{~min}$. After a survival period of 3-5 d, rats were deeply anesthetized and perfused as above. Coronal sections containing the injection sites and the thalamus were cut at $50 \mu \mathrm{m}$ on a vibratome, cryoprotected, and processed with $\mathrm{ABC}$ using $\mathrm{Ni}-\mathrm{DAB}$ as a substrate.

\section{Results}

\section{Size and topography of receptive fields}

Delimited laterally by the ventral lateral posterior nucleus (VPL), VPMvl is a crescent-shaped region that approximately corresponds to the lower tier of the VPM. It is thicker caudally and thins out rostrolaterally. Thus, to sample a reasonable number of VPMvl cells per descent, the recording electrode was lowered through the caudolateral aspect of the VPM, where vibrissae of rows $\mathrm{A}$ and $\mathrm{B}$ are represented. Figure $1 A$ illustrates the distribution of whisker-sensitive cells sampled in five vertical electrode penetrations in that region of the VPM. Starting dorsally, $\sim 4.5$ $\mathrm{mm}$ below the surface of the brain, the first vibrissa-sensitive units were encountered, most of which responded briskly to a single vibrissa. Yet, in the most medial descents (descents 4, 5), some of the first encountered units responded to multiple whiskers (see Discussion). As the recording electrode advanced through the VPM, units were driven by a single whisker pertaining to rows $\mathrm{A}$ or $\mathrm{B}$. Then, a series of multiwhisker cells were 
A
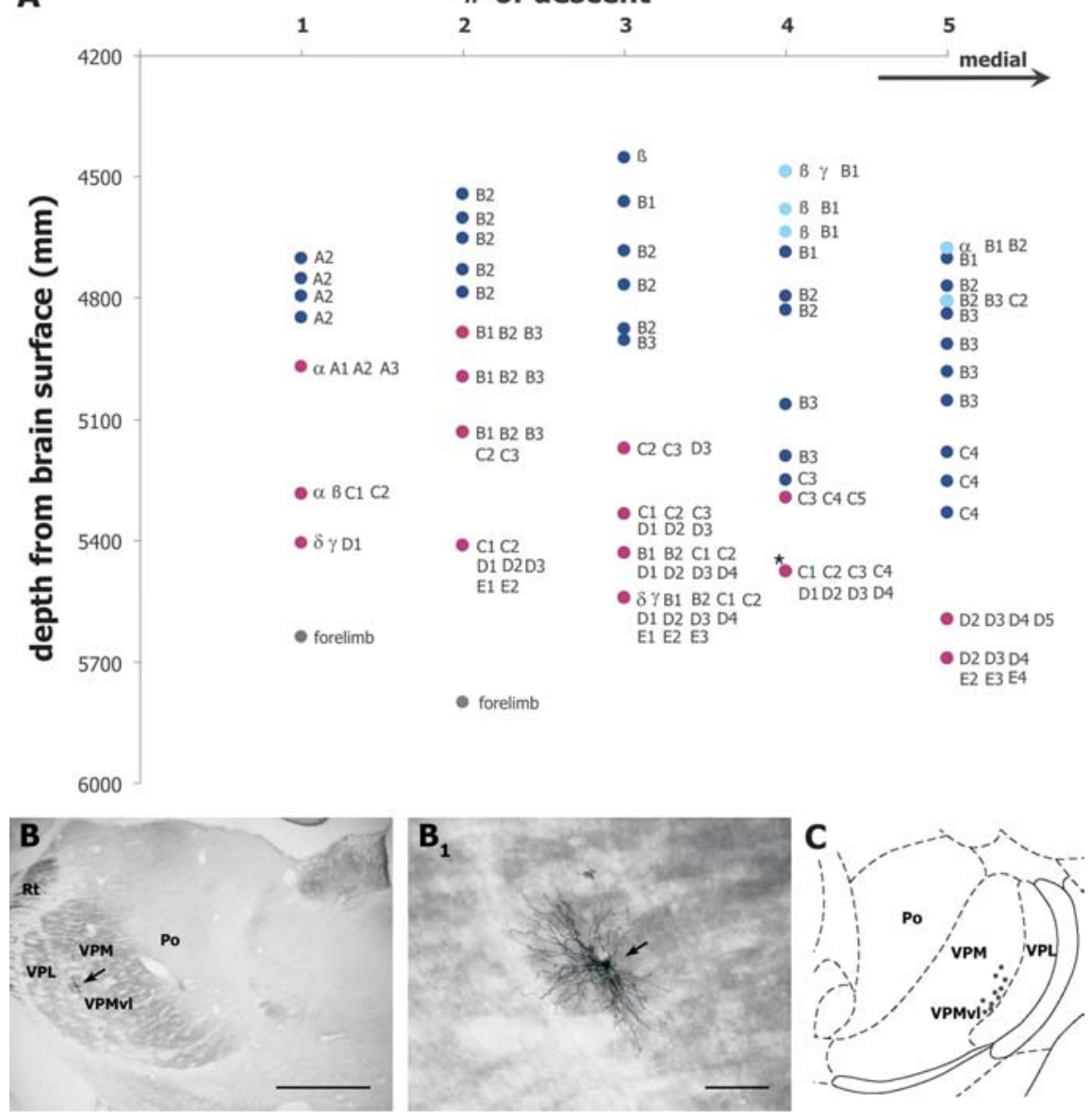

Figure 1. Receptive field size of vibrissa-sensitive units in the somatosensory thalamus. $\boldsymbol{A}$, Receptive field size of cells recorded along five vertical electrode tracks in the VPM in a rat (dark blue dots, monowhisker cells; light blue dots, multiwhisker cells in dorsal VPM; purple dots, multiwhisker cells in VPMvl; gray dots, cells that responded to stimulation of the forelimb). Vibrissae within the receptive field of each unit are identified next to each dot. $\boldsymbol{B}$, VPMvl cell labeled with Neurobiotin (arrow). The location of this unit is indicated by an asterisk in $\boldsymbol{A}$ (tract 4 ), and the same cell is shown at higher magnification in $\boldsymbol{B}_{1}$. C, Location of 10 labeled multiwhisker cells on a representative coronal section of the thalamus $(\sim 3.8 \mathrm{~mm}$ behind the bregma) (drawing modified from Paxinos and Watson, 1998). Scale bars: $\boldsymbol{B}, 1 \mathrm{~mm} ; \boldsymbol{B}_{1}, 100 \mu \mathrm{m}$.

recorded before the electrode entered into the VPL, where cells responded to stimulation of the forelimb or neck. A similar clustering of multiwhisker units was observed in the VPMvl of all rats $(n=9)$, which was confirmed by the juxtacellular labeling of at least one unit in each of the experiments $(n=10$ cells) (Fig. $1 B, C)$.

As estimated with a hand-held probe or controlled deflection of individual vibrissae VPMvl cells responded to several whiskers (mean receptive field size, $7.16 \pm 2.75$ vibrissae; range, $4-13 ; n=$ 53 cells; 17 cells tested with the piezoelectric stimulator). Receptive field topography exhibited a slight preference for row over arc of whisker representation. The number of vibrissae within the longest row divided by that in the longest arc in receptive fields yielded a ratio of $1.37 \pm 0.48$, a ratio inferior to that reported for the multiwhisker cells in the SpVic $(1.87 \pm 0.58)$ (Furuta et al., 2006). In our sample of VPMvl cells no clear principal whisker was identified, for most units responded with near equal magnitude to two to four whiskers (see Fig. 3B).

\section{Effect of PrV lesion}

To determine the potential contribution of $\operatorname{PrV}$ afferents to receptive field size in VPMvl, vibrissal responses were also assessed after lesion of the PrV in 3 rats. Parasagittal brainstem lesions were made medial to the $\operatorname{PrV}$, so that the nucleus remained intact while ascending crossing axons were destroyed (Fig. 2A). As estimated from CO-stained sections of the brainstem, lesions extended rostrocaudally between frontal planes $8-10.5$ behind the bregma and throughout the depth of the brainstem. The lesion severed crossing axons that arise from the $\operatorname{PrV}$ and part of the PrV itself, but the descending branches of primary afferents that travel in the trigeminal tract remained intact (Fig. $2 A$ ). In each lesioned rat, recordings were first performed before the lesion to locate the VPMvl. Then, the PrV was lesioned, and the receptive field of the units in the same thalamic region was mapped. As expected, the vast majority of VPM cells failed to respond to whisker deflection after the lesion, but VPMvl units kept responding to multiple whiskers. The location of latter units was confirmed by juxtacellular labeling. The mean receptive field size in lesioned rats was $7.08 \pm 2.27$ whiskers ( $n=12$ units), which is not significantly different from the mean receptive field size found in intact animals (7.16 \pm 2.75 whiskers; $n=53$ units, unpaired $t$ test; $p=0.91$ ). As estimated by the mean number of spikes per deflection, no significant difference in response magnitude was found between normal and lesioned rats $(1.31 \pm 0.84, n=17$; and $1.56 \pm 0.68, n=12$, respectively; unpaired $t$ test; $p=0.21$ ). Likewise, the mean onset latency of responses to controlled whisker deflection did not significantly differ between cells recorded in normal and lesioned rats $(8.43 \pm 2.13 \mathrm{~ms}, n=17$; and $8.2 \pm 2.26 \mathrm{~ms}, n=12$, respectively; unpaired $t$ test; $p=0.46$ ) (Fig. $2 B, C$ ). In sum, lesion experiments confirm that receptive field synthesis in the VPMvl is independent of lemniscal inputs, and therefore that it relies primarily on inputs from the SpVic.

\section{Angular tuning and direction preference}

It was reported previously that SpVic cells exhibit sensitivity to the direction of vibrissa deflection (Furuta et al., 2006). We thus explored the extent to which VPMvl responses reflect the directional tuning of their presynaptic partners. Figure $3 A, B$ shows bar graphs and normalized polar plots comparing the directional tuning of the responses elicited by the vibrissae composing the receptive field of two VPMvl cells. For each vibrissa within the receptive field of a cell (17 cells, 55 vibrissae), a directionselectivity index, $D$, was calculated as a measure of directional tuning (Taylor and Vaney, 2002). $D$ was defined as $D=V i / r i$, where Vi are vector magnitudes pointing in the direction of the stimulus and having length, $r i$, equal to the number of spikes recorded during that stimulus. $D$ can range from 0 , when the responses are equal in all stimulus directions, to 1 , when a response is obtained only for a single stimulus direction. Thus, values for $D$ approaching 1 indicate asymmetric responses over a small range of angles and therefore sharper directional tuning. 


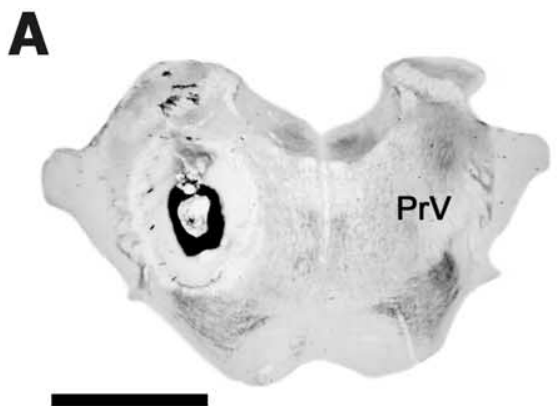

Br. $-9.3 \mathrm{~mm}$

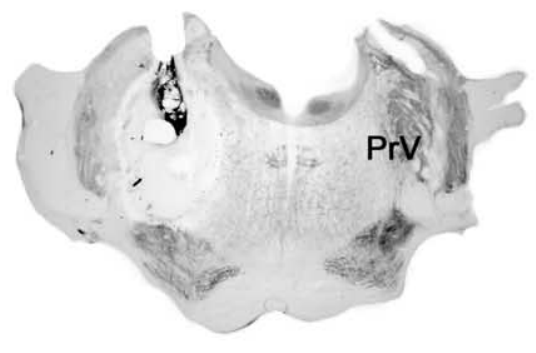

Br. $-10.0 \mathrm{~mm}$

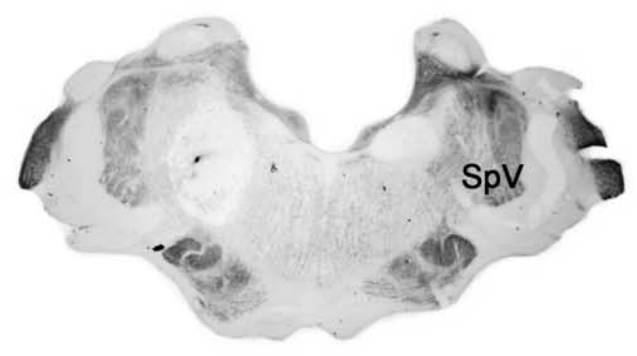

Br. $-10.5 \mathrm{~mm}$
B

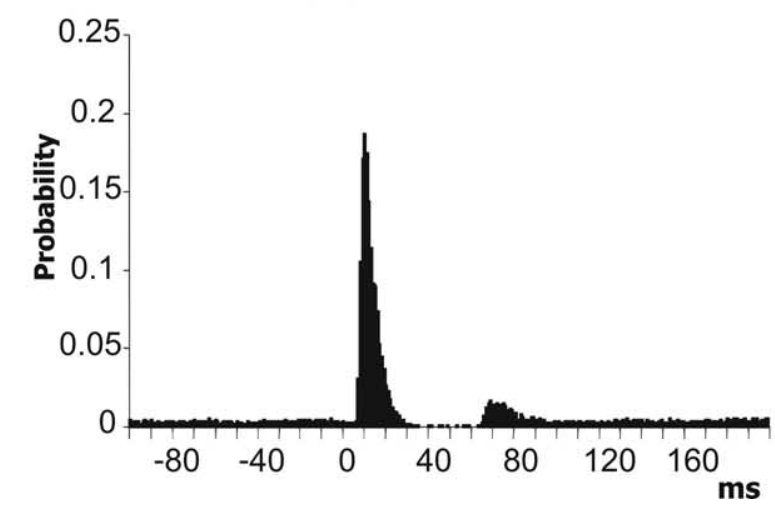

C
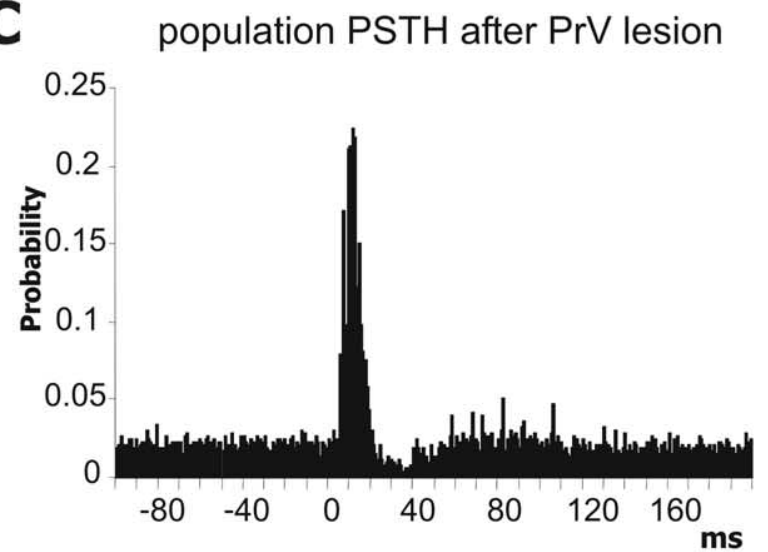

Figure 2. Effect of PrV lesion on receptive field size of VPMvl units. $\boldsymbol{A}$, Extent of an electrolytic lesion that severed ascending axons from the PrV. The frontal plane of each costained section is indicated below (Br, bregma). $\boldsymbol{B}, \boldsymbol{C}$, Population PSTHs of vibrissal responses in normal and lesioned rats, respectively (sum of all responses to all directions; 18 cells in $\boldsymbol{B}, 12$ cells in $\boldsymbol{C}$ ). The higher level of background activity in $\boldsymbol{C}$ was caused by juxtacellular current that was applied to assess the presence of inhibition in some cells. Prestimulus background activity in $\boldsymbol{B}$ and $\boldsymbol{C}$ was subtracted from the poststimulus counts to compare the magnitude and latency of responses. Scale bar, $3 \mathrm{~mm}$.

The mean $D$ value in VPMvl was $0.22 \pm 0.17$, which indicates a lower degree of angular tuning than that measured in the SpVic $(0.47 \pm 0.34)$ (Furuta et al., 2006).

The multivibrissa structure of receptive fields in the VPMvl raises the issue of whether the direction of motion that elicits the largest response is the same for all the vibrissae in the receptive field of a cell. To address this question, we first built polar plots that were normalized with respect to the largest response evoked by the most effective vibrissa, and computed the vector sum of individual polar plots. Then, by computing the vector sum of all vibrissa-associated vectors within the receptive field of a cell, a grand vector was obtained that represented the ensemble direction tuning of that cell. The consistency of direction preference was estimated by computing the absolute value of the difference in angle between each of the vibrissa-associated vectors and the grand vector. The median value of angular difference was $38^{\circ} \pm$ 82.15 , which indicates a lower consistency than in the SpVic (a median difference of $23^{\circ}$ was computed in the SpVic) (Furuta et al., 2006). The polar graph of Figure $3 C$ shows the distribution of grand vectors whose length corresponds to the normalized magnitude of angular preference among a population of $17 \mathrm{VPMvl}$ cells. This graph reveals a broad range in the overall degree of angular tuning and, like in the SpVic, an absence of angular tuning preference for any particular direction.

In brief, the metrics of vibrissal responses in VPMvl reveal that cells have large receptive field that includes nearly as many vibrissae within row and arc, and exhibit a lower degree of direction selectivity than in the SpVic.

\section{Reticular thalamic and corticothalamic projections to the VPMvl}

Previous anatomical studies have shown that the thalamic nuclei that serve as relay stations in the lemniscal and paralemniscal pathways received innervation from specific sets of RT and corticothalamic neurons (Bourassa et al., 1995; Pinault et al., 1995; Crabtree, 1999; Désîlets-Roy et al., 2002; Killackey and Sherman, 2003). We thus examined whether a similar specificity of feedback projections prevailed in the VPMvl.

Reticular thalamic cells derive their receptive field from thalamic relay cells. In deeply anesthetized animals the vast majority of vibrissa-sensitive reticular cells respond to a single whisker and, as a rule, their axon terminates in the corresponding barreloid (Désîlets-Roy et al., 2002). Because VPMvl cells respond to several whiskers, one would expect some reticular cells to respond to multiple whiskers and to send projection to the VPMvl. Among the 158 whisker-responsive reticular units recorded in 10 rats, six responded to the deflection of multiple vibrissae (3-7 vibrissae) at a mean latency of $11.21 \pm 2.45 \mathrm{~ms}$ (Fig. $4 \mathrm{~A}$, population PSTH). These cells were clustered in the lateral wedge of the nucleus beneath the monowhisker units (Fig. $4 B$ ). The juxtacellular labeling of five multiwhisker reticular cells confirmed that they innervated principally the VPMvl (Fig. 4C-F). Figure $4 D-F$ shows horizontal, coronal and sagittal views of a fully reconstructed cell. After leaving the reticular thalamic nucleus, the axon ran caudally and medially up to the posterior group (Po) border, where it gave off thin collaterals bearing few terminals. Then, the axon divided into thick branches that descended 


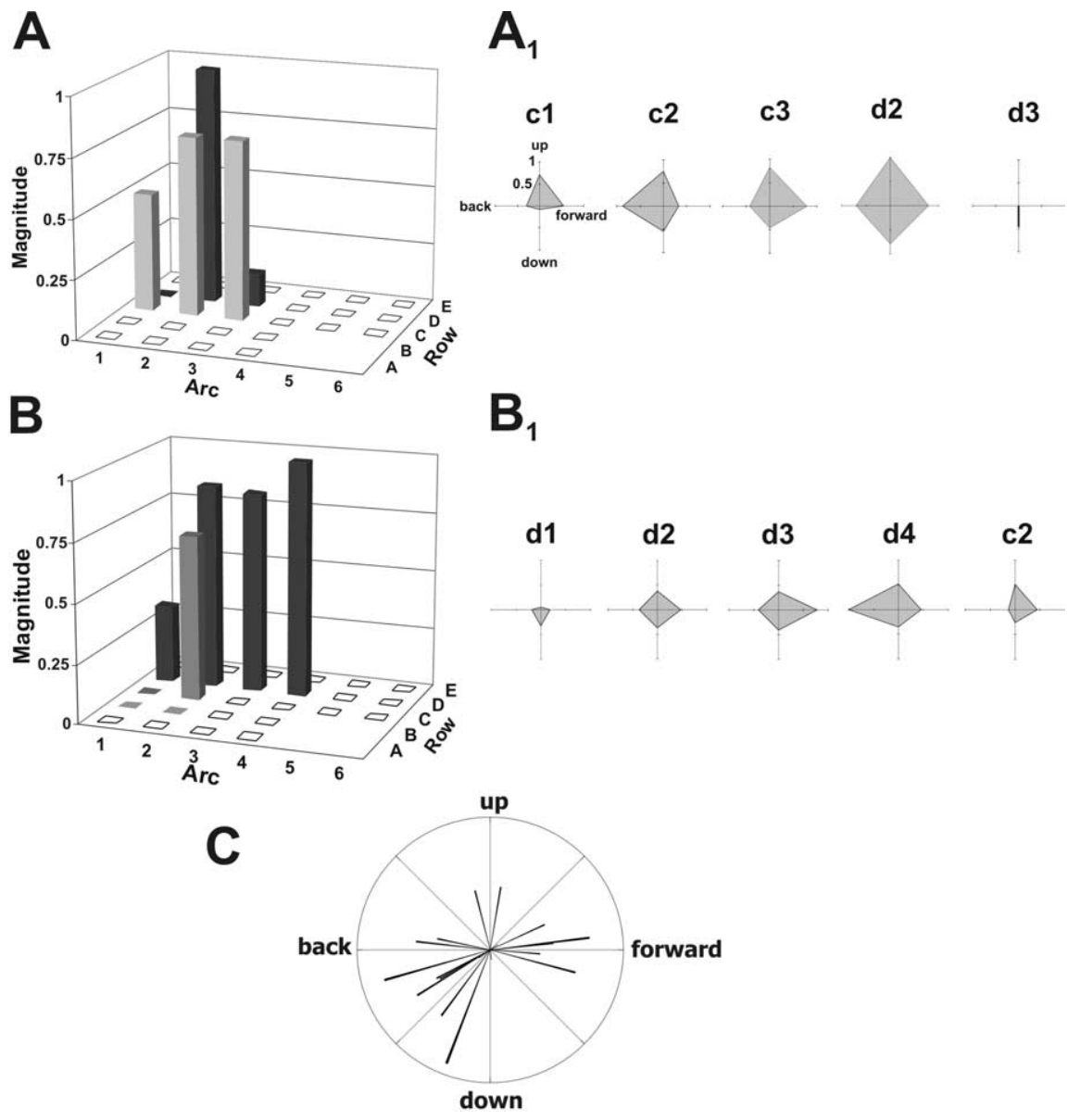

Figure 3. Response properties and directional tuning of multivibrissa VPMvl cells. $\boldsymbol{A}, \boldsymbol{B}$, Three-dimensional bar graphs of normalized response magnitudes within the receptive field of two VPMvl cells. For each vibrissa, response magnitudes were summed across all directions. Bars with zero magnitude (B1 and $\mathrm{C} 1$ in the bottom graph, for example) indicate vibrissae of a threshold too high to be tested with the piezo stimulator. Polar plots in A1 and A2 show normalized response magnitudes for the same two units after deflection of each vibrissa in four cardinal directions. C, Angular tuning preference of VPMvl cells. Each vector is the vector sum of all vibrissa-associated vectors within the receptive field of a cell (17 cells). Vector length corresponds to the normalized magnitude of angular preference among a population of 17 VPMvl cells.

through the VPM and arborized in the VPMvl, where the terminal field spread rostrocaudally over a large expanse of the nucleus (rostrocaudal extent, $\sim 700 \mu \mathrm{m}$; diameter, $\sim 200 \mu \mathrm{m}$ ). A similar topography characterized the terminal field of the other multiwhisker cells that were labeled in the reticular nucleus.

Because VPMvl relay cells project to S2 and to the dysgranular zone of S1 (Pierret et al., 2000), one would expect these cortical areas to return projections to the VPMvl. FluoroGold injection into the VPMvl led to the retrograde labeling of lamina 6 cells in the granular and dysgranular zones across the whole extent of S1 and in S2, and also in the transition zone between S2 and the insular and auditory cortices (Fig. 5A-E). Few retrogradely labeled neurons were found in layer 5 in S1, and none in S2. Injection sites also involved part of the overlying barreloids so that, on the basis of this result, one cannot decide on the prevalence of the CT cells in S1 that project solely to the VPMvl. Yet, retrograde tracer injection into the barreloids was never reported to produce retrograde labeling in S2, suggesting that S2 returns projection to the VPMvl in a highly specific manner. This was further confirmed by the topographic distribution of terminal fields after anterograde tracer injection in the infragranular layers of S2 (two rats) (Fig. $6 H$ ). Terminal labeling was observed in Po and in the VPMvl, which also contained some retrogradely labeled cell bod- ies (Fig. 6A-G,I,J). The rest of the VPM contained labeled fibers en route toward Po, but terminals were notably absent. Like corticothalamic axons that arise from lamina 6 cells in the other areas of the cerebral cortex, those issuing from S2 divided into thin branches that were covered with arrays of drumstick-like boutons (Fig. $6 K$ ).

\section{Discussion}

The VPMvl was initially identified as a distinct region of the VPM after tract tracing experiments showed that this thalamic territory receives vibrissal input from the SpVic (Pierret et al., 2000). Except for the absence of barreloids, which are difficult to detect in the adult rodent, no cytoarchitectonic feature or immunohistochemical stain exist that delineate the boundary of VPMvl within the VPM. The situation is even more ambiguous in the caudal part of the thalamus where the VPMvl and Po territories merge into a region that receives input from both the rostral and caudal parts of the SpVi (Williams et al., 1994). Despite these anatomical uncertainties, the present study provides clear evidence that the VPMvl serves as a distinct thalamic relay station for vibrissal messages. This pathway differs from the classic lemniscal pathway, in that VPMvl cells exhibit large multiwhisker receptive fields that are independent of input from the PrV, and receive afferents from specific populations of RT and corticothalamic neurons.

\section{Receptive field properties of VPM and VPMvl neurons}

There is general consensus that the receptive field size of VPM neurons depends on the depth of anesthesia (Friedberg et al., 1999; Aguilar and Castro-Alamancos, 2005). Yet, this dependence seems to apply primarily to the relay cells within the core of barreloids, which receive input from small-sized cells in the PrV whose receptive field is reduced to a single whisker in deeply anesthetized animals. In contrast and just like SpVic projection cells, VPMvl neurons maintain large receptive field under deep anesthesia (Furuta et al., 2006). In addition, no principal whisker dominated their receptive field and their response was insensitive to PrV lesion. This clearly demonstrates different modes of integration of vibrissal inputs in pathways that pass through the barreloids and VPMvl.

The dorsalmost part of the barreloids also contains cells that maintain multiwhisker receptive field under deep anesthesia (Urbain and Deschênes, 2007). These cells however receive their driving input from a subpopulation of multiwhisker cells in the $\mathrm{PrV}$, not from the SpVi. Thus, because barreloids form curved, obliquely oriented rods with respect to the vertical, different ratios of monowhisker and multiwhisker cells can be obtained in vertical electrode descents through the VPM in deeply anesthetized animals. In penetrations aiming the VPMvl (i.e., in the lateral aspect of the VPM) multiwhisker cells in the head of the barreloids can be missed, or their number underestimated. This 
A

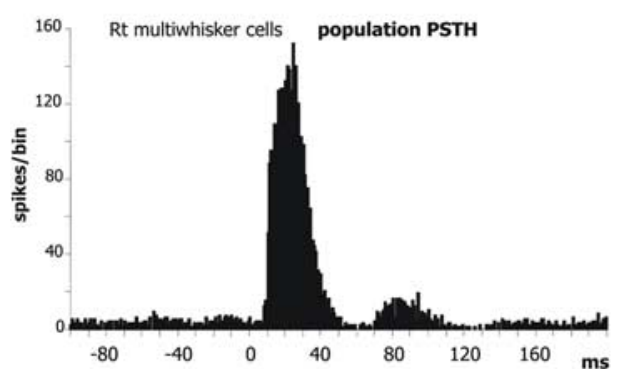

D

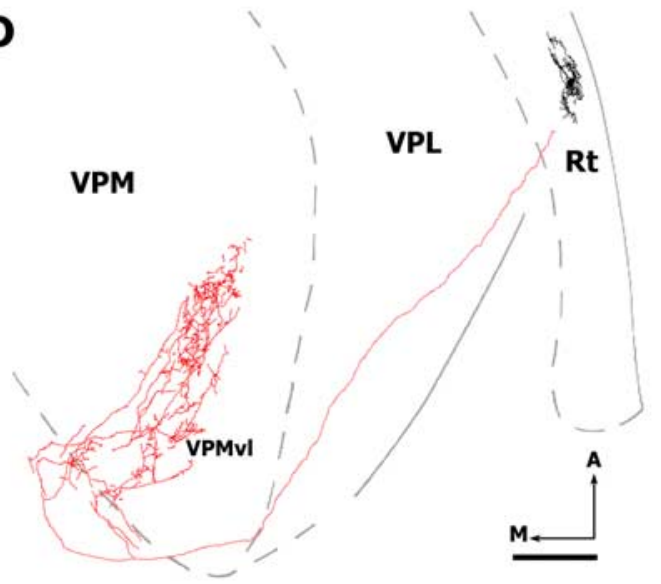

$\mathbf{F}$

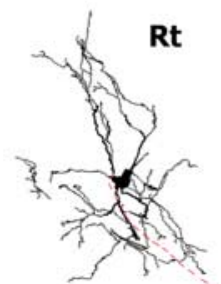

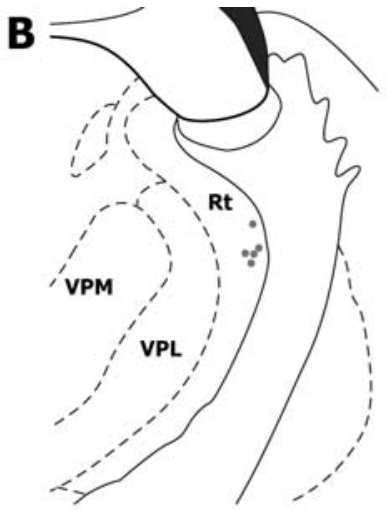

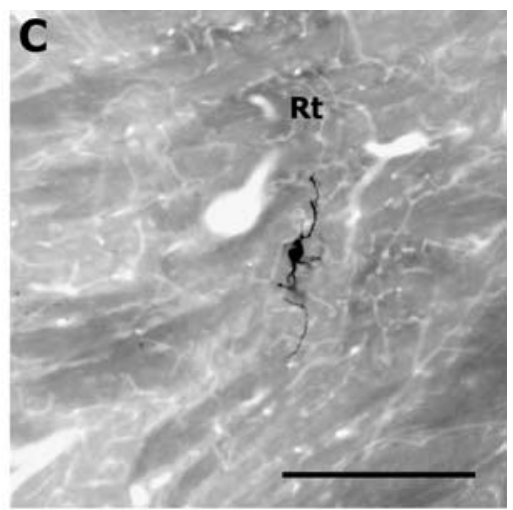

E
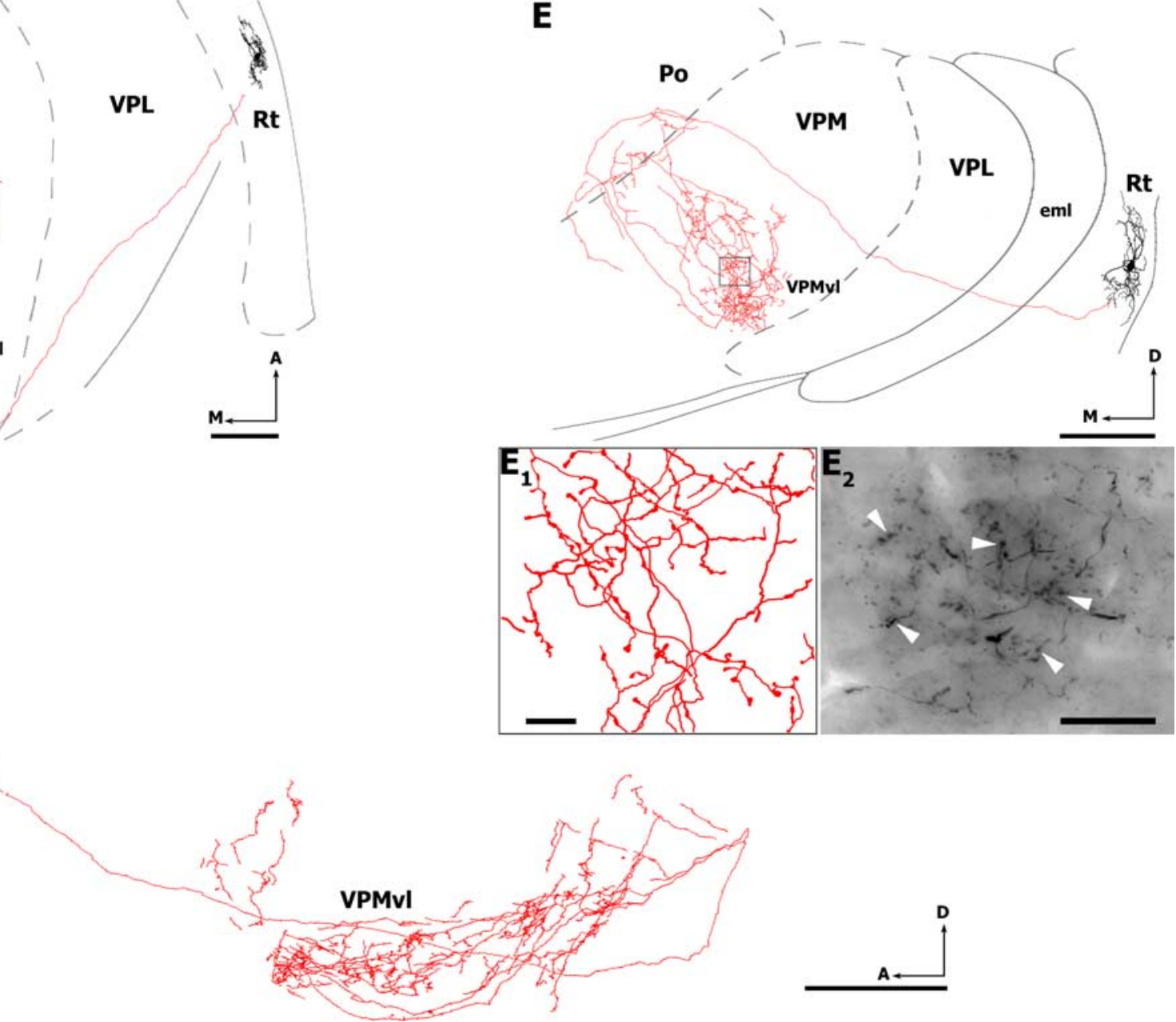

Figure 4. Axonal projections of multivibrissa RT cells. $\boldsymbol{A}$, Population PSTH of vibrissal responses recorded in six multiwhisker units (sum of all responses to all directions). Dots in $\boldsymbol{B}$ indicate the location of five multiwhisker RT units labeled with Neurobiotin. The RT cell labeled in $\boldsymbol{C}$ was reconstructed from serial sections, and drawings in $\boldsymbol{D}-\boldsymbol{F}$ show horizontal $(\boldsymbol{D})$, coronal $(\boldsymbol{E})$, and sagittal $(\boldsymbol{F})$ views of the dendritic and axonal arbors. Note that the axonal arbor is mostly restricted to the VPMvl, with a few collaterals in Po. $\boldsymbol{E}_{1}$, Axonal branches and boutons (arrowheads) within the framed area in $\boldsymbol{E}$. Photomicrograph in $\boldsymbol{E}_{\mathbf{2}}$ show a cluster of corticothalamic boutons in the VPMvl (arrowheads). A, Anterior; $\boldsymbol{D}$, dorsal; eml, external medullary lamina; $\boldsymbol{M}$, medial. Scale bars: $\boldsymbol{B}, 1 \mathrm{~mm} ; \boldsymbol{C} \boldsymbol{F}$, $200 \mu \mathrm{m} ; \boldsymbol{E}_{1}, 10 \mu \mathrm{m} ; \boldsymbol{E}_{2}, 25 \mu \mathrm{m}$.

is reflected in our results by the absence of multiwhisker cells in dorsal VPM when electrodes were lowered in the lateral aspect of the nucleus, and the presence of some multiwhisker cells in dorsal VPM along more medial electrode descents (Fig. 1C).

Our data indicate that VPMvl responses, albeit similar in several respects to those of SpVic cells, differ markedly from their presynaptic counterparts along a number of parameters, suggesting that the receptive field structure and response properties of individual VPMvl neurons derive from converging input of a small number of SpVic neurons. According to Furuta et al. (2006), the receptive field of SpVic projection neurons comprises, on average, $4.8 \pm 3.3$ vibrissae, which is significantly smaller than the receptive field size presently found in the VPMvl (7.16 \pm 2.75 vibrissae). This difference likely relies on two factors: (1) the data of Furuta et al. (2006) included a number of cerebellar projecting cells that have smaller receptive field than thalamic projecting cells (Woolston et al., 1982); (2) compared with the small size of the terminal field of PrV axons in a barreloid (diam- 

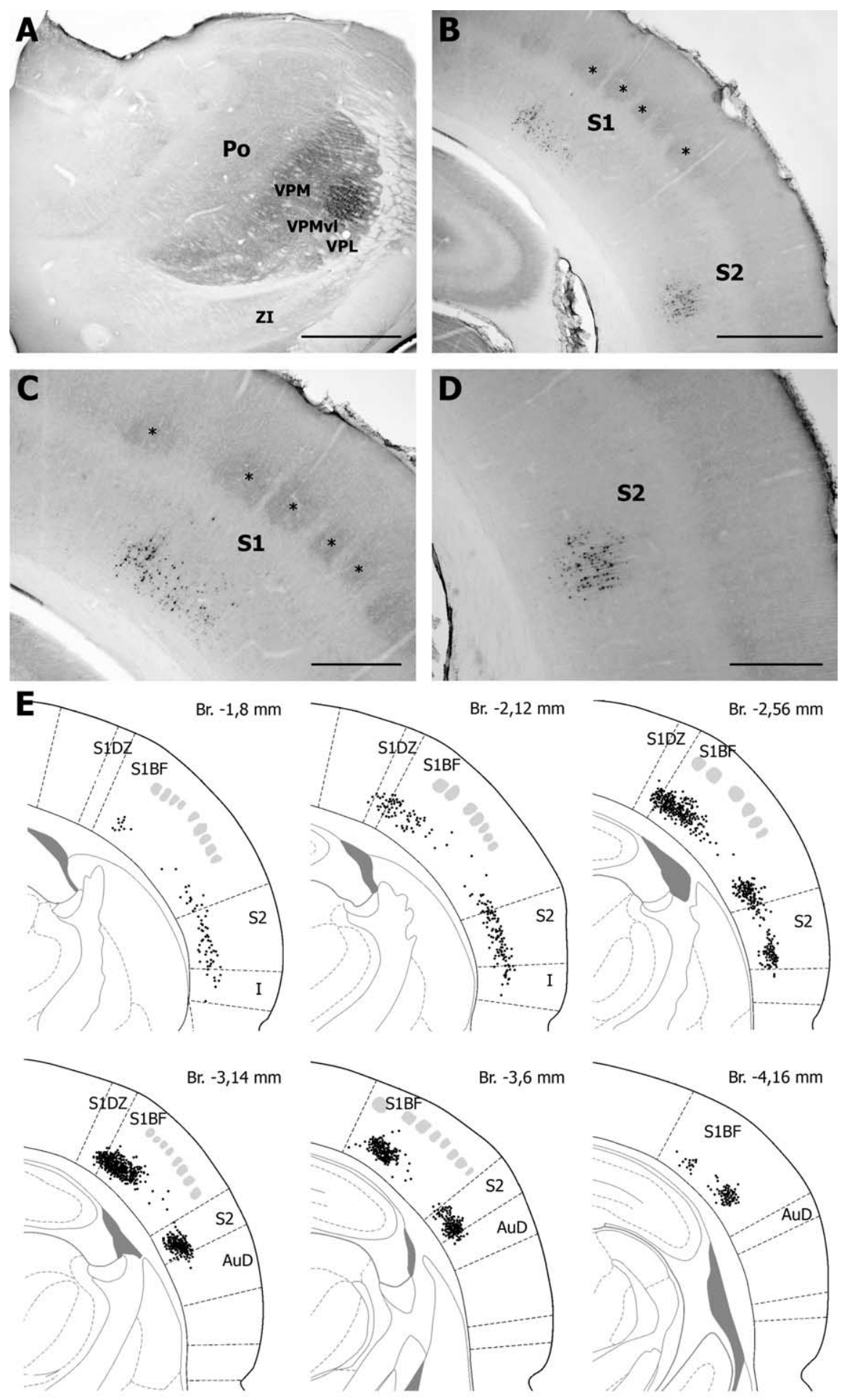

Figure 5. Retrograde labeling in the cerebral cortex after FluoroGold injection in the VPMvl. The injection site shown in $A$ led to retrograde labeling of layer 6 cells in S1 and S2 (B). Clusters of labeled cells in S1 and S2 are shown at higher magnification in $C$ and $\boldsymbol{D}$, respectively. Asterisks indicate coreactive barrels. $\boldsymbol{E}$, Distribution of retrogradely labeled cells in $\mathbf{S 1}$ and $\mathbf{S 2}$ on representative sections taken at different frontal planes (drawings adapted from Paxinos and Watson, 1998) [distance from the bregma (Br) as indicated; gray patches, barrels]. AuD; Dorsal auditory cortex; I, insular cortex; S1BF, barrel field of S1; S1DZ, dysgranular zone of S1; Zl, zona incerta. Scale bars: $A, B, 1 \mathrm{~mm} ; \boldsymbol{C}, \boldsymbol{D}, 500 \mu \mathrm{m}$.

eter $\sim 80 \mu \mathrm{m})$ (Veinante and Deschênes, 1999), individual SpVic axons form larger, rostrocaudally oriented terminal fields in VP$\operatorname{Mvl}($ size $\sim 100 \times 250 \mu \mathrm{m})$ (Veinante et al., 2000). Because the density of relay cells and trigeminal terminals are similar in the two compartments of VPM (H. Bokor and L. Acsády, unpub- lished observations), input convergence likely contributes to enlarge receptive field size in VPMvl. The convergence of SpVic inputs may also explain the lower degree of angular tuning and the low consistency in directional preference among the different whiskers that compose the receptive field of a cell.

A point that still remains unclear is whether PrV and SpVic inputs converge on cells that straddle the transition zone between the core of the barreloids and the VPMvl. This possibility was suggested by the labeling study of Pierret et al. (2000), who found cells in the transition region that projected to both a barrel and to the adjoining dysgranular zone. Moreover, a double labeling electron microscopy study reported that boutons from $\mathrm{PrV}$ and $\mathrm{SpVi}$ contact the same VPM neuron (Wang and Ohara, 1993). Yet, that study did not provide any detail on the prevalence of converging synapses nor on the location of recipient cells in the VPM. Despite these uncertainties, the convergence of $\mathrm{PrV}$ and SpVic axons on some cells seems likely, because there is no evidence that the dendrites of barreloid and VPMvl relay cells are spatially segregated in the transition zone between VPMvl and the overlying barreloids.

\section{Reticular thalamic and corticothalamic loops}

If one defines a sensory pathway as a threeneuron chain that links a sensory organ to a specific area of the neocortex, then the vibrissal system of rodents comprises at least four parallel pathways of information processing (summarized by Urbain and Deschênes, 2007). Each of these pathways arises from a specific population of brainstem trigeminal neurons, transits through a different thalamic region, and has its own areal and laminar distribution in the neocortex. Moreover, for each of the relay stations in the thalamus, specific sets of corticothalamic and RT projections have been proposed. The present study provides anatomical evidence that a similar organization plan prevails in the VPMvl, which receives specific inputs from corticothalamic cells in S2 and from a subpopulation of multiwhisker RT neurons.

A methodological constraint in our study is that FG injection aimed at the VP$\mathrm{Mvl}$ also involved the VPL and part of the overlying barreloids. However, corticothalamic projections to the barreloids were shown to arise from upper lamina 6 cells in barrel columns (Bourassa et al., 1995; Killackey and Sherman, 2003). Thus, the presence of retrogradely labeled cells in lamina 6 of the septal columns of S1 and in S2 clearly indicates that both S1 and S2 contribute to VPMvl inner- 
vation. Thus, the dual origin of corticothalamic projections complies with the rule of reciprocity of connections between cortex and thalamus because individual VPMvl axons project to both the dysgranular zone of S1 and to S2 (Pierret et al., 2000).

\section{Barrel- and septa-related cortical circuits}

Because VPMvl cells respond to the deflection of multiple whiskers, cortical cells that receive input from VPMvl should exhibit multiwhisker receptive fields that are independent of the cortical circuitry. Recordings in anesthetized rats indeed reported that S2 and septal neurons have large receptive fields (Armstrong-James et al., 1992; Brumberg et al., 1999; Kwegyir-Afful and Keller, 2004). Moreover, latency analyses of vibrissal responses in barrel cortex indicates that septal neurons respond to whisker stimuli at virtually the same time as barrel neurons, but several milliseconds before supragranular neurons (Armstrong-James et al., 1992; Brumberg et al., 1999), which indicates that both septal and barrel cells must derive their receptive field input from the thalamus.

The rodent somatosensory cortex contains barrel-related and septa-related circuits that represent two processing streams that differ in their response patterns and anatomical connections (for review, see Alloway, 2008). Whereas the barrel-related circuits process lemniscal inputs that arises from the VPM, the septarelated circuits are often considered to process paralemniscal inputs conveyed through Po. However, Po can hardly be responsible for the vibrissal response of septal cells in anesthetized animals, because sensory transmission through this higher-order nucleus is normally impeded by feedforward inhibition that arises from the zona incerta (Trageser and Keller, 2004; Lavallée et al., 2005). Thus, septal cells might derive their multiwhisker responses from the VPMvI or from the recently discovered multiwhisker pathway that passes through the head of the barreloids (Urbain and Deschênes, 2007). In the latter case, one would expect a lesion of the PrV to render barrel and septal cells unresponsive to whisker deflection.

\section{References}

Aguilar JR, Castro-Alamancos MA (2005) Spatiotemporal gating of sensory inputs in thalamus during quiescent and activated states. J Neurosci 25:10990-11002.

Alloway KD (2008) Information processing streams in rodent barrel cortex: the differential functions of barrels and septal circuits. Cereb Cortex 18:979-989.

Armstrong-James M, Fox K, Das-Gupta A (1992) Flow of excitation within rat barrel cortex on striking a single vibrissa. J Neurophysiol 68:1345-1358.

Bourassa J, Pinault D, Deschênes M (1995) Corticothalamic projections from the cortical barrel field to the somatosensory thalamus in rats: a
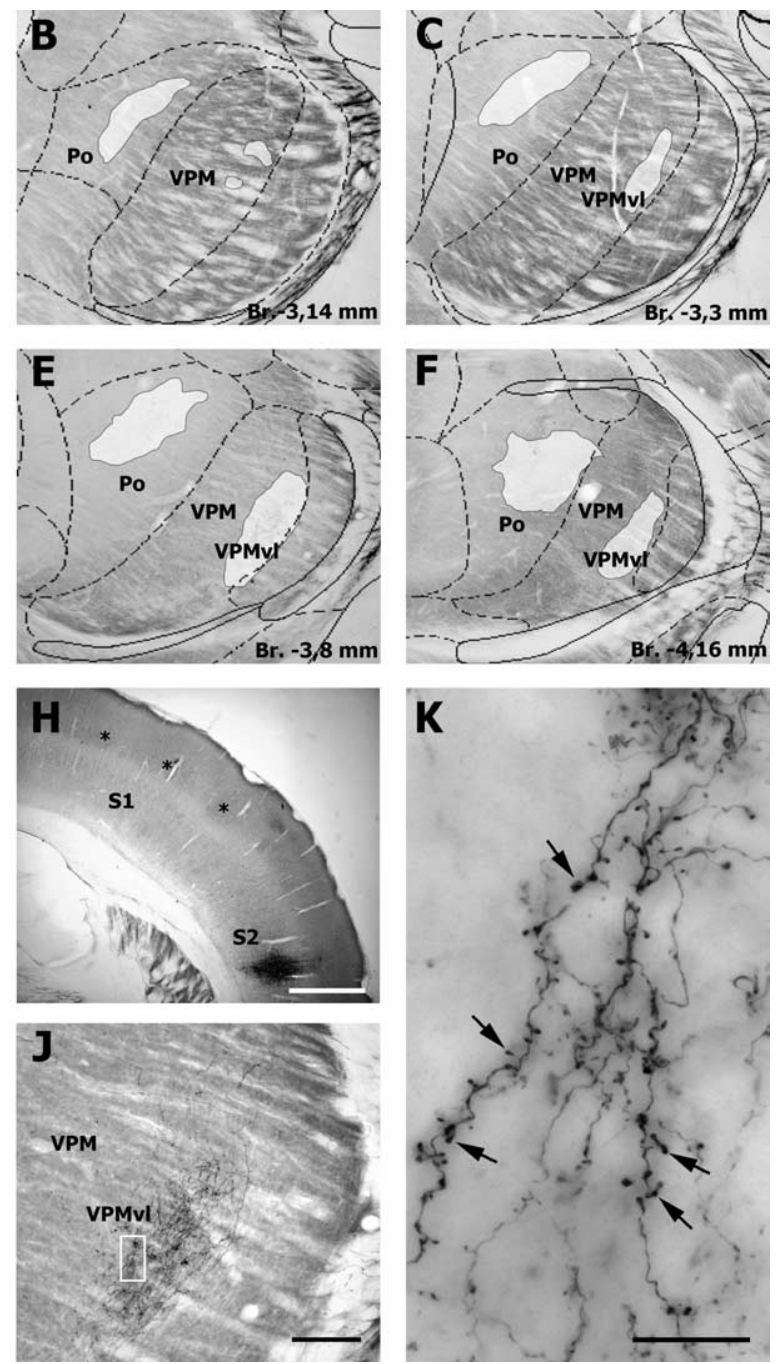

Figure 6. Corticothalamic cells in S2 project to the VPMvl. $\boldsymbol{A}-\mathbf{G}$, Distribution of corticothalamic terminals (white patches) in . Note that corticothalamic terminals form two separate clusters: one in Po and the other one in VPMvl (arrows framed area in $J$. Note the drumstick like boutons (arrows) given offen passant by the fine branches of corticothalamic axons. Ang, Angular thalamic nucleus; Br., bregma. Scale bars: $\boldsymbol{A}-\boldsymbol{I}, 500 \mu \mathrm{m} ; \boldsymbol{H}, 1 \mathrm{~mm} ; \boldsymbol{J}, 200 \mu \mathrm{m} ; \boldsymbol{K}, 20 \mu \mathrm{m}$.

single-fibre study using biocytin as an anterograde tracer. Eur J Neurosci 7:19-30.

Brumberg JC, Pinto DJ, Simons DJ (1999) Cortical columnar processing in the rat whisker-to-barrel system. J Neurophysiol 82:1808-1817.

Crabtree JW (1999) Intrathalamic sensory connections mediated by the thalamic reticular nucleus. Cell Mol Life Sci 56:683-700.

Deschênes M, Veinante P, Zhang Z-W (1998) The organization of corticothalamic projections: reciprocity versus parity. Brain Res Rev 28:286-308.

Désîlets-Roy B, Varga C, Lavallée P, Deschênes M (2002) Substrate for cross-talk inhibition between thalamic barreloids. J Neurosci 22:RC218(1-4).

Friedberg MH, Lee SM, Ebner FF (1999) Modulation of receptive field properties of thalamic somatosensory neurons by the depth of anesthesia. J Neurophysiol 81:2243-2252.

Furuta T, Nakamura K, Deschênes M (2006) Angular tuning bias of vibrissa-responsive cells in the paralemniscal pathway. J Neurosci 26:10548-10557.

Ito M (1988) Response properties and topography of vibrissa-sensitive VPM neurons in the rat. J Neurophysiol 60:1181-1197.

Killackey HP, Sherman SM (2003) Corticothalamic projections from the rat primary somatosensory cortex. J Neurosci 23:7381-7384.

Kim U, Ebner FF (1999) Barrels and septa: separate circuits in rat barrels field cortex. J Comp Neurol 408:489-505. 
Kwegyir-Afful EE, Keller A (2004) Response properties of whisker-related neurons in rat second somatosensory cortex. J Neurophysiol 92:2083-2092.

Lavallée P, Urbain N, Dufresne C, Bokor H, Acsády L, Deschênes M (2005) Feedforward inhibitory control of sensory information in higher-order thalamic nuclei. J Neurosci 25:7489-7498.

Minnery BS, Bruno RM, Simons DJ (2003) Response transformation and receptive-field synthesis in the lemniscal trigeminothalamic circuit. J Neurophysiol 90:1556-1570.

Paxinos G, Watson W (1998) The rat brain in stereotaxic coordinates, Ed 4. London: Academic

Pierret T, Lavallée P, Deschênes M (2000) Parallel streams for the relay of vibrissal information through thalamic barreloids. J Neurosci 20:7455-7462.

Pinault D (1996) A novel single-cell staining procedure performed in vivo under electrophysiological control: morpho-functional features of juxtacellularly labeled thalamic cells and other central neurons with biocytin or Neurobiotin. J Neurosci Methods 65:113-136.

Pinault D, Bourassa J, Deschênes M (1995) The axonal arborization of single thalamic reticular neurons in the somatosensory thalamus of the rat. Eur J Neurosci 7:31-40.

Rhoades RW, Belford GR, Killackey HP (1987) Receptive field properties of rat ventral posterior medial neurons before and after selective kainic acid lesions of the trigeminal brain stem complex. J Neurophysiol 57:1577-1600.

Shepherd GM, Svoboda K (2005) Laminar and columnar organization of ascending excitatory projections to layer $2 / 3$ pyramidal neurons in rat barrel cortex. J Neurosci 25:5670-5679.

Sugitani M, Yano J, Sugai T, Ooyama H (1990) Somatotopic organization and columnar structure of vibrissae representation in the rat ventrobasal complex. Exp Brain Res 81:346-352.

Taylor WR, Vaney DI (2002) Diverse synaptic mechanisms generate direction selectivity in the rabbit retina. J Neurosci 22:7712-7720.

Trageser JC, Keller A (2004) Reducing the uncertainty: gating of peripheral inputs by zona incerta. J Neurosci 24:8911-8915.

Urbain N, Deschênes M (2007) A new thalamic pathway of vibrissal information modulated by the motor cortex. J Neurosci 27:12407-12412.

Van der Loos H (1976) Barreloids in the mouse somatosensory thalamus. Neurosci Lett 2:1-6.

Varga C, Sík A, Lavallée P, Deschênes M (2002) Dendroarchitecture of relay cells in thalamic barreloids: a substrate for cross-whisker modulation. J Neurosci 22:6186-6194.

Veinante P, Deschênes M (1999) Single- and multi-whisker channels in the ascending projections from the principal trigeminal nucleus in the rat. J Neurosci 19:5085-5095.

Veinante P, Jacquin MF, Deschênes M (2000) Thalamic projections from the whisker-sensitive regions of the spinal trigeminal complex in the rat. J Comp Neurol 420:233-243.

Wang BR, Ohara PT (1993) Convergent projections of trigeminal afferents from the principal nucleus and subnucleus interpolaris upon rat ventral posteromedial thalamic neurons. Brain Res 629:253-259.

Williams MN, Zahm DS, Jacquin MF (1994) Differential foci and synaptic organization of the principal and spinal trigeminal projections to the thalamus in the rat. Eur J Neurosci 6:429-453.

Woolston DC, La Londe JR, Gibson JM (1982) Comparison of response properties of cerebellar- and thalamic-projecting interpolaris neurons. J Neurophysiol 48:160-173.

Yu C, Derdikman D, Haidarliu S, Ahissar E (2006) Parallel thalamic pathways for whisking and touch signals in the rat. PLoS Biol 4:e124. 